\section{Endoscopic ultrasound-guided ERCP in the treatment of a Todani type-III cyst causing acute necrotizing pancreatitis}

Bile duct cysts are defined as dilatations of the extrahepatic or intrahepatic biliary tree. Biliary cysts are classified according to the Todani classification, which encompasses five types. They account for approximately $1 \%$ of all benign biliary disease. The incidence of biliary cysts in Europe is $1-2$ per 1000000 . The Todani type-III cyst is rare and accounts for $2 \%-$ $4 \%$ of all Todani cysts. The risk of developing a cholangiocarcinoma ranges from $2.5 \%$ to $28 \%$, and increases with age. Choledocholithiasis, cholelithiasis, hepatolithiasis, cholangitis, pancreatitis, and spontaneous cyst perforation have all been reported in these patients [1,2].

We report the case of a 17-year-old Latino man with systemic candidosis occurring after acute necrotizing pancreatitis (ANP) that was caused by a choledochal cyst. The patient was referred to our unit 35 days after two failed attempts at endoscopic retrograde cholangiopancreatography (ERCP). The patient also had right kidney agenesis.

On arrival, the patient's clinical condition was critical owing to sepsis and cholestasis. A computed tomography (CT) scan was performed, which showed the presence of multiple stones inside the choledochal cyst with a dilated common bile duct (CBD). The pancreatic isthmus and tail contained necrotic areas. An endoscopic ultrasound (EUS) was performed, which showed that the cyst was completely full of stones and occluding the common bile duct.
Using a duodenoscope, we performed a cystotomy, opening the cyst with a needle knife. The stones were then totally removed ( Video 1 ). The patient developed bleeding from the cystotomy 3 days later, which was successfully managed using endoscopic hemostasis. Although the bleeding was controlled, he died the following day from multiorgan failure and systemic sepsis.

Acute necrotizing pancreatitis is a lifethreatening condition, especially for patients in a poor clinical condition. Endoscopic cystotomy is a possible treatment for choledochal Todani type-III cysts, especially in patients who have undergone an unsuccessful attempt at ERCP. This treatment has not been previously reported.

Endoscopy_UCTN_Code_TTT_1AR_2AI

\section{Competing interests: None}

\section{Benedetto Mangiavillano,} Andrea Parodi, Massimo Conio

Gastroenterology \& Gastrointestinal Endoscopy, General Hospital, Sanremo, Italy

\section{References}

1 Cerwenka H. Bile duct cyst in adults: interventional treatment, resection, or transplantation? World J Gastroenterol 2013; 19: 5207-5211

2 Jabłońska B. Biliary cysts: etiology, diagnosis and management. World J Gastroenterol 2012; 18: 4801 - 4810

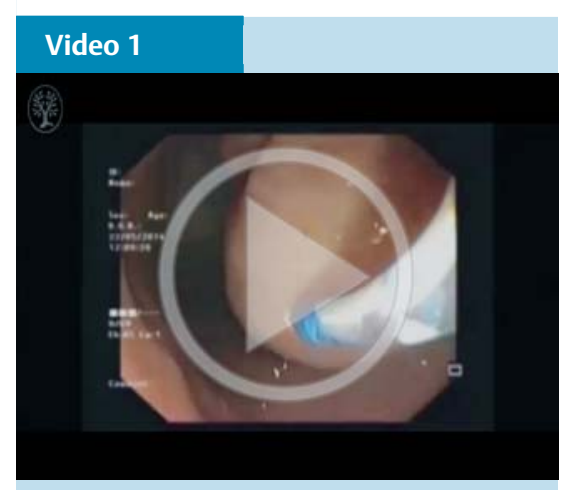

Endoscopic ultrasound (EUS) and endoscopic views of the choledochal cyst. Cystotomy is performed with a needle knife, revealing evidence of multiple stones in the opened cyst.

Bibliography

DOI http://dx.doi.org/

10.1055/s-0042-100201

Endoscopy 2016; 48: E44

(c) Georg Thieme Verlag KC

Stuttgart · New York

ISSN 0013-726X

\section{Corresponding author}

Benedetto Mangiavillano, MD

Gastroenterology Gastrointestinal Endoscopy

Borea Hospital - Sanremo

Via G. Borea 56

18038 Sanremo

Italy

Fax: +39-0184-536875

b_mangiavillano@hotmail.com 